\title{
Effects and molecular mechanisms of Achyranthes bidentata polypeptide $k$ on proliferation of Schwann cells
}

\author{
Leili Tang ${ }^{1 \#}$, Min Zhang ${ }^{1 \#}$, Xingyu Liu' ${ }^{2}$, Ye Zhu ${ }^{3}$, Xin Chen ${ }^{4}$, Jingfei Zhong ${ }^{5}$, Meiyuan Li ${ }^{1}$
}

${ }^{1}$ Key Laboratory of Neuroregeneration of Jiangsu, Ministry of Education, NMPA Key Laboratory for Research and Evaluation of Tissue Engineering Technology Products, Jiangsu Clinical Medicine Center of Tissue Engineering and Nerve Injury Repair, Co-Innovation Center of Neuroregeneration, Nantong University, Nantong, China; ${ }^{2}$ School of Medicine, Nantong University, Nantong, China; ${ }^{3}$ Academy of Medical Engineering and Translational Medicine, Tianjin University, Tianjin, China; ${ }^{4}$ Department of Neurology, Affiliated Hospital of Nantong University, Nantong, China; ${ }^{5}$ Nantong University Xinglin College, Nantong, China

Contributions: (I) Conception and design: M Li; (II) Administrative support: None; (III) Provision of study materials or patients: M Zhang, X Liu, Y Zhu, X Chen, J Zhong; (IV) Collection and assembly of data: L Tang; (V) Data analysis and interpretation: L Tang, M Zhang; (VI) Manuscript writing: All authors; (VII) Final approval of manuscript: All authors.

\#These authors contributed equally to this work.

Correspondence to: Meiyuan Li. Key Laboratory of Neuroregeneration of Jiangsu, Ministry of Education, NMPA Key Laboratory for Research and Evaluation of Tissue Engineering Technology Products, Jiangsu Clinical Medicine Center of Tissue Engineering and Nerve Injury Repair, CoInnovation Center of Neuroregeneration, Nantong University, 19 Qixiu Road, Nantong 226001, China. Email: limeiyuan1986@ntu.edu.cn; Jingfei Zhong. Nantong University Xinglin College, Nantong 226001, China. Email: zjfkaixin@ntu.edu.cn.

Background: Achyranthes bidentata polypeptide k (ABPPk) is an active ingredient separated from the Achyranthes bidentata polypeptides (ABPP) in traditional Chinese medicine. In the present study, we investigated the promoting effects and molecular mechanisms of $\mathrm{ABPPk}$ on the proliferation of Schwann cells (SCs).

Methods: Primary SCs were cultured with ABPPk or nerve growth factor (NGF) in vitro, and cell viability, cell cycle, EdU assay, and the expressions of proliferating cell nuclear antigen (PCNA) and Ki67 were analyzed. In addition, RNA-seq was used for bioinformatics analysis at different time points. PCNA was detected at different time points in a rat sciatic nerve injury model to further determining the role of $\mathrm{ABPPk}$ in sciatic nerve injury repair.

Results: We found that ABPPk could effectively promote the proliferation of SCs, while ABPPk and NGF had different molecular mechanisms for their proliferation at different time points. Weighted gene coexpression network analysis (WGCNA) showed that ABPPk was mainly involved in the positive regulation of cell proliferation and epigenetic regulation of cell proliferation, while the main cell proliferation-related modules that NGF participated in were attenuation of negative regulation of cell proliferation and positive regulation of cell cycle. There were significant differences in the genes involved in different modules between the two groups, and $\mathrm{ABPPk}$ differed from NGF in the biological process of SC migration, differentiation, movement, and development in terms of action time and key genes. Functional enrichment analysis revealed $\mathrm{ABPPk}$ had more advantages and participation in the axon extension and vascular system areas. Furthermore, $\mathrm{ABPPk}$ significantly promoted the proliferation of SCs in vivo.

Conclusions: Through in vitro and in vivo studies, we identified the promoting effects of ABPPk on the proliferation of SCs. Using high-throughput sequencing technology, our work more comprehensively revealed the characteristics and mechanism of $\mathrm{ABPPk}$ on SCs. These results further enrich an understanding of the positive function and molecular mechanism of $\mathrm{ABPPk}$ in peripheral nerve regeneration and are conducive to the discovery of new therapeutic targets for peripheral nerve regeneration.

Keywords: Achyranthes bidentata polypeptide k (ABPPk); cell proliferation; Schwann cells (SCs); molecular mechanism; peripheral nerve injury 
Submitted Aug 22, 2021. Accepted for publication Oct 16, 2021.

doi: $10.21037 /$ atm-21-5181

View this article at: https://dx.doi.org/10.21037/atm-21-5181

\section{Introduction}

The repair of peripheral never injury is both extremely common in clinic and a complicated biological process. Due to the slow regeneration of nerves, tissue adhesion and muscle atrophy, the functional recovery of damaged nerves is always limited. Current research on the regeneration of peripheral nerves mainly focuses on the promotion of axonal growth and the remodeling of neural function with the recovery of anatomical structure $(1,2)$. As an essential glial cell in the peripheral nervous system, Schwann cells (SCs) play a crucial role in axon regeneration (3-5). After peripheral nerve injury, proliferating SCs can secrete a variety of neurotrophic factors to promote axon growth and myelin sheath formation $(3,6)$. The promotion of SCs would greatly benefit the treatment of peripheral nerve damage and the improvement of neurological disease, and is a hot topic in research on peripheral nerve regeneration and repair.

Chinese traditional medicine is an important part of the country's culture, with the advantages of abundant resources, affordable prices, and low side effects. Achyranthes bidentate is a significant medical plant that can promote blood circulation and strengthen muscles and bones, which has aroused widespread research interest in the medical field $(7,8)$. In recent years, Achyranthes bidentata polypeptide k (ABPPk) purified by high-performance liquid chromatography (HPLC) from Achyranthes bidentata polypeptides (ABPP) has been shown to provide excellent protective efficiency to neurons (9), and in our previous study, we further explored whether it had a significant effect on oxidative damage to SCs. The results showed ABPPk could inhibit the apoptosis of SCs, and was closely related to the two signal pathways of PI3K/AKT and ERK1/2 (10). In view of the important role of SCs in the pathogenesis of peripheral nerve disease, their proliferation, migration, and apoptosis have been extensively studied, although current research is limited to the effects of a few genes or microRNAs (miRNAs). There are few studies on certain Chinese medicines that can promote the proliferation of SCs, and a lack of comprehensive and systematic basic research on the entire proliferation process of SCs $(11,12)$. Therefore, exploring effective ways to activate and promote
SC proliferation has important value for the repair of peripheral nerve injury, and provides new targets for its clinical treatment.

This study investigated whether $\mathrm{ABPPk}$ promoted SC proliferation and observed it significantly did so in vitro and in vivo, indicating significant potential for peripheral nerve regeneration and repair. To investigate the molecular mechanism of ABPPk on SCs, transcriptomic data of ABPPk treated group and nerve growth factor (NGF) treated group were analyzed by bioinformatics methods. Our work demonstrates the correlation and difference of ABPPk and NGF on SCs at the molecular regulatory, respectively. These results also contribute to a comprehensive and accurate understanding of the molecular mechanisms underlying the effect of ABPPk on SCs, which may help to explore the therapeutic targets of peripheral nerve injury. We present the following article in accordance with the ARRIVE reporting checklist (available at https:// dx.doi.org/10.21037/atm-21-5181).

\section{Methods}

\section{Ethical statement}

This study was performed under a project license (No. 20190303-15) granted by the Laboratory Animal Ethics Committee of Nantong University, in compliance with Nantong University institutional guidelines for the care and use of animals.

\section{Preparation of $A B P P k$}

The ABPPk used in the experiment was independently isolated and extracted as described previously (13). Before use, ABPPk was diluted with Dulbecco's Modified Eagle Medium (DMEM) for cell experiments, and with saline for in vivo experiments.

\section{Cell treatment}

SCs were obtained from the sciatic nerve of SpragueDawley (SD) rats $1 \mathrm{~d}$ after birth, and primary cultured in vitro. Rats were acquired from the Experimental Animal 
Center of Nantong University [license No. SCXK (Su) 2014-0001 and SYXK (Su) 2012-0031, No. 20190225-004]. The culture method used has been previously described (14). In brief, cells were cultured in DMEM medium (Corning, USA) supplemented with $10 \%$ of fetal bovine serum (FBS; Gibco, Grand Island, NY, USA), then anti-Thy1.1 antibody (Sigma, St. Louis, MO, USA) and rabbit complement (Invitrogen, Carlsbad, CA, USA) were added to remove fibroblasts. Purified SCs were identified by immunostaining and plated in 96- or 6-well plates, with the culture medium replaced by DMEM medium with $10 \%$ of FBS containing $0.5 \mu \mathrm{g} / \mathrm{mL}$ ABPPk or $0.1 \mu \mathrm{g} / \mathrm{mL}$ NGF, respectively. Multiple time points (15 min, 0.5, 1, 2, 3, 6, 12, and $24 \mathrm{~h}$ ) were selected for detection and analysis.

\section{Cell counting kit-8 (CCK-8) assay}

Cell proliferation was monitored by the CCK-8 assay (Dojindo, Kumamoto, Japan) following the manufacturer's protocol. Briefly, the SCs were plated at a density of $3 \times 10^{3}$ cells/well on 96-well plates and subjected to different treatments. Ten $\mu \mathrm{L}$ of CCK-8 solution was added and incubated for $2 \mathrm{~h}$ at $37^{\circ} \mathrm{C}$, and the absorbance was determined at $450 \mathrm{~nm}$ using a Microplate reader (Bio-tek Instruments, Inc.).

\section{Cell cycle analysis}

Cells were harvested and fixed in ice-cold $70 \%$ ethanol overnight at $4{ }^{\circ} \mathrm{C}$. After washing twice with phosphatebuffered saline (PBS), they were then incubated with propidium iodide (PI; Sigma-Aldrich, St. Louis, MO, USA) in the dark for $30 \mathrm{~min}$, and analyzed by flow cytometry (BD Biosciences, San Jose, CA, USA).

\section{EdU assay}

Following designated treatment, the proliferation of SCs was quantified by Cell-LightTM EdU DNA Cell Proliferation Kit (Ribobio, Guangzhou, China) according to the manufacturer's protocol. Nuclei were counterstained using Hoechst 33342, and the percentage of EdU-positive cells was captured on a DMR fluorescence microscope (Leica Microsystems, Bensheim, Germany). The procedures above were conducted in triplicate.

\section{Western blot analysis}

Total protein was separated by $10 \%$ sodium dodecyl sulfate-polyacrylamide gel electrophoresis (SDS-PAGE) and transferred to polyvinylidene fluoride (PVDF) membranes (Millipore, Bedford, MA, USA). The membranes were blocked with $5 \%$ nonfat milk for $2 \mathrm{~h}$ at $37^{\circ} \mathrm{C}$, then incubated with the primary antibodies Rabbit anti-proliferating cell nuclear antigen (PCNA; 1:1,000; Abcam) and mouse anti-GAPDH (1:5,000; Abcam) at $4{ }^{\circ} \mathrm{C}$ overnight. After washing three times, secondary antibodies were incubated for $1 \mathrm{~h}$ at room temperature. The Odyssey densitometry program (LI-COR, Lincoln, NE, USA) was used to detect the signals and GAPDH was used as the reference protein.

\section{Immunofluorescence staining}

Cells cultured on coverslips were fixed with $4 \%$ paraformaldehyde for $30 \mathrm{~min}$ at room temperature. After washing with PBS twice and blocked for $90 \mathrm{~min}$, they were then stained with anti-Ki67 (1:200; Abcam) and anti-S100 (1:200; Abcam) at $4{ }^{\circ} \mathrm{C}$ overnight, followed by Goat antiRabbit IgG-Cy3 (1:200; Invitrogen) and Donkey antiMouse IgG-Alex-488 (1:200; Invitrogen). Finally, the cells were stained with Hoechst 33342 or DAPI, and analyzed with a fluorescence microscopy (AxioImager M2, Zeiss).

\section{RNA-seq analysis}

Samples at $15 \mathrm{~min}, 0.5,1,2,3,6,12$, and $24 \mathrm{~h}$ with three duplicates of three different groups (ABPPk, NGF and normal SCs) were collected. According to the manufacturer's protocols, the 75 RNA samples were extracted, and RNA integrity was determined using the Agilent 2100 Bioanalyzer (Agilent Technologies, USA). Strand-specific RNA-seq libraries were constructed, and the samples were sequenced on the Illumina Hiseq $\mathrm{X}$ Ten sequencing platform (OEbiotech, Shanghai, China). All subsequent analyses were performed using clean reads. The sequencing data were uploaded to the sequence read archive (SRA) database (accession number: PRJNA764187).

\section{Weighted gene co-expression network analysis (WGCNA)}

To investigate co-expression gene modules across time points in SCs with ABPPk or NGF, we performed WGCNA. Power $\beta=7$ was selected to calculate an adjacency matrix, then an "unsigned" network was inferred in a manner of one-step network construction. Expression patterns of genes 
within given modules were visualized using a pheatmap package implemented in $\mathrm{R}$, and functional enrichment of genes within a given module was conducted using the online DAVID database.

\section{Bioinformatic analysis}

In addition to the proliferation of SCs, we also analyzed the possible mechanisms of differentially expressed genes in the biological processes of SC migration, differentiation, movement, and development. Ingenuity Pathway Analysis (IPA; Ingenuity System, Redwood City, CA, USA) was used to perform bioinformatics analysis. Genes with expression changes were uploaded to the IPA software and compared and analyzed with those related to these functions obtained in IPA. Heatmap analysis data that graphically represents values in color were also used. The expression values of the differentially expressed genes were analyzed for hierarchical clustering. The Search Tool for the Retrieval of Interacting Genes (STRING; http://string.embl.de/) was used to construct a proteinprotein interaction (PPI) network which was visualized by Cytoscape (http://www.cytoscape.org/) (15).

\section{Functional enrichment analysis}

The $\mathrm{R}$ package clusterProfiler process allows gene classification and calculation of enrichment for gene ontology (GO) terms (16), and adjusted $\mathrm{P}<0.05$ was considered to indicate statistically significant enriched function annotations.

\section{Preparation of rat sciatic nerve defect model}

The Experimental Animal Center of Nantong University provided SD rats weighing 180-200 g, and which lived at $24{ }^{\circ} \mathrm{C}$ and $55 \% \pm 5 \%$ humidity and cycled in light/dark for $12 \mathrm{~h}$. After all animals were deeply anesthetized with ketamine/xylaxine, the sciatic nerve was dissected carefully, and a $3 \mathrm{~mm}$-long-nerve section was crushed three times (10 s each time with $10 \mathrm{~s}$ interval) with a hemostatic forceps. The crush cite was marked with an 8-0 nylon suture, and the animals were randomly divided into three groups with six rats in each group. The rats were subjected to subepineural microinjection at the injury site with $\mathrm{ABPPk}$ in $0.5 \mathrm{mg} / \mathrm{mL}$, NGF in $0.1 \mathrm{mg} / \mathrm{mL}$, and saline respectively. The tissues of the clamped sections were taken for detection on the 1, 4, and $7 \mathrm{~d}$ after the operation.

\section{Statistical analysis}

Results are presented as means \pm standard error of the mean (SEM) and the statistical significance was conducted by Student's $t$-tests for comparing two groups or oneway analysis of variance (ANOVA) for three and more comparisons. $\mathrm{P}<0.05$ was considered to indicate a statistically significant difference.

\section{Results}

\section{Effect of ABPPk on the viability of SCs}

Immunocytochemistry with anti-S100 antibody serving as SC markers provided evidence of the cell purity (Figure 1A), and the CCK- 8 method was used to observe the effects of ABPPk treatment at different times on SC viability. SCs were treated with $0.5 \mu \mathrm{g} / \mathrm{mL} \mathrm{ABPPk}$ and $0.1 \mu \mathrm{g} / \mathrm{mL}$ NGF for different periods of time, and complete medium (DMEM with $10 \% \mathrm{FBS}$ ) was used as the control group. The cell viability was tested for different culture times $(15 \mathrm{~min}, 0.5$, $1,2,3,6,12$, and $24 \mathrm{~h}$ ), and it was found that ABPPk and NGF treatment for different times had different degrees of effect on the viability of SCs (Figure 1B).

\section{Effect of ABPPk on cell cycle progression of SCs}

The cell cycle assay was analyzed by flow cytometry, and showed the number of cells in the G1/G0 phase was reduced, while that in the $\mathrm{S}$ phase was significantly increased compared to the control, and was especially obvious at 2, 3, 6, and $12 \mathrm{~h}$ (Figure 2).

\section{Effect of ABPPk on Edu of SCs}

To determine whether ABPPk increased proliferation of SCs, we observed cells labeled with EdU under a fluorescence microscope. This showed ABPPk significantly increased $\mathrm{EdU}$ incorporation, and the cell proliferation was most obvious at $15 \mathrm{~min}, 1,3,6$, and $12 \mathrm{~h}(\mathrm{P}<0.05)$ (Figure 3).

\section{Effect of ABPPk on the expression levels of PCNA and Ki67}

We further analyzed cell proliferation markers using Western blot and immunocytochemistry, and the results showed the expression levels of PCNA were upregulated after treatment with ABPPk (Figure 4). In addition, the Ki67 staining analysis indicated a higher Ki67 positive cell 
A

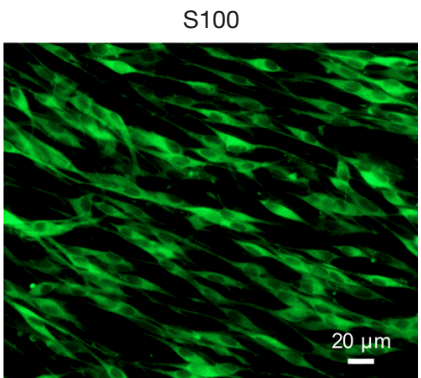

DAPI

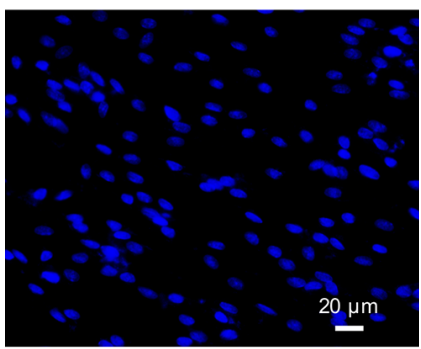

Merge

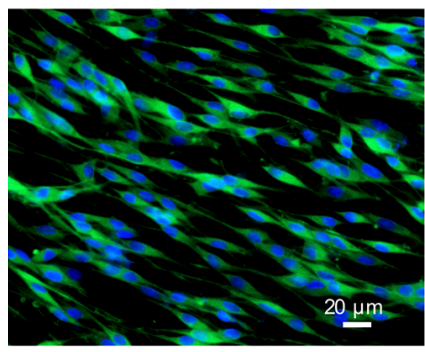

B

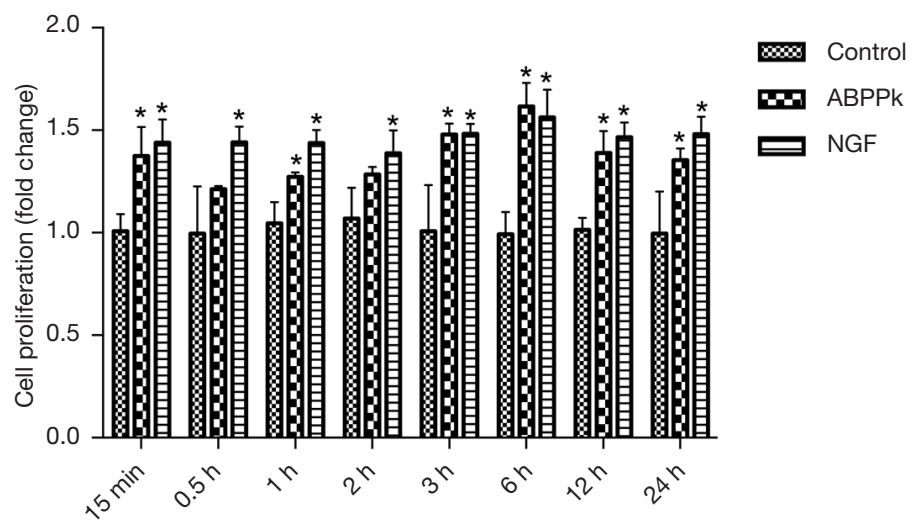

Figure 1 Effect of ABPPk on viability of SCs. (A) Fluorescent immunocytochemistry of cultured SCs stained by S100 (green) with nuclei counterstained with DAPI (blue). Scale bar $=20 \mu \mathrm{m}$. (B) SCs were incubated with $0.5 \mu \mathrm{g} / \mathrm{mL}$ ABPPk or $0.1 \mu \mathrm{g} / \mathrm{mL} \mathrm{NGF} \mathrm{for} \mathrm{the} \mathrm{indicated}$ times (15 min, 0.5, 1, 2, 3, 6, 12, and $24 \mathrm{~h}$ ), and the viability of SCs was measured by CCK-8 assay. *, $\mathrm{P}<0.05$ vs. the control cells. ABPPk, Achyranthes bidentata polypeptide k; SCs, Schwann cells; NGF, nerve growth factor; CCK-8, cell counting kit-8.

proportion at $15 \mathrm{~min}, 3,6$, and $12 \mathrm{~h}$ in the $\mathrm{ABPPk}$ group, while no significant increase was observed at $0.5,1,2$, and $24 \mathrm{~h}(\mathrm{P}<0.05)$ (Figure 5). Taken together, the results show $\mathrm{ABPPk}$ can promote the proliferation of SCs.

\section{Identification of hub genes in cell proliferation related modules}

To overview the expression patterns of DEGs in ABPPk and NGF groups, many modules analyzed by WGCNA were observed. In the module of positive regulation of cell proliferation in the $\mathrm{ABPPk}$ group, there were 116 genes, which were mainly involved in promoting cell proliferation, with the highest expression in the middle time period. Another module related to cell proliferation in the ABPPk group was the epigenetic regulation of cell proliferation, the trend of which was closer to the module of positive regulation of cell proliferation. There were also modules related to cell proliferation in the NGF group, which were the two modules of attenuation of negative regulation of cell proliferation and positive regulation of cell cycle, with the trends also relatively close (Figure 6). There were many genes involved in the four modules, and there were significant differences in the genes involved in different modules between the group of ABPPk and NGF (Figures S1-S4).

\section{Identifying the DEGs of SCs with different functions and bub genes from the PPI network in the ABPPk group}

The IPA database platform was used to search the biological processes of migration, differentiation, movement, and development of SCs. The molecular networks at different time points were further linked by heatmap to show the dynamic changes of molecular regulatory networks of different biological functions (Figure 7). The PPI networks of hub genes with high connectivity in different functions of SCs were screened (Figure S5). 

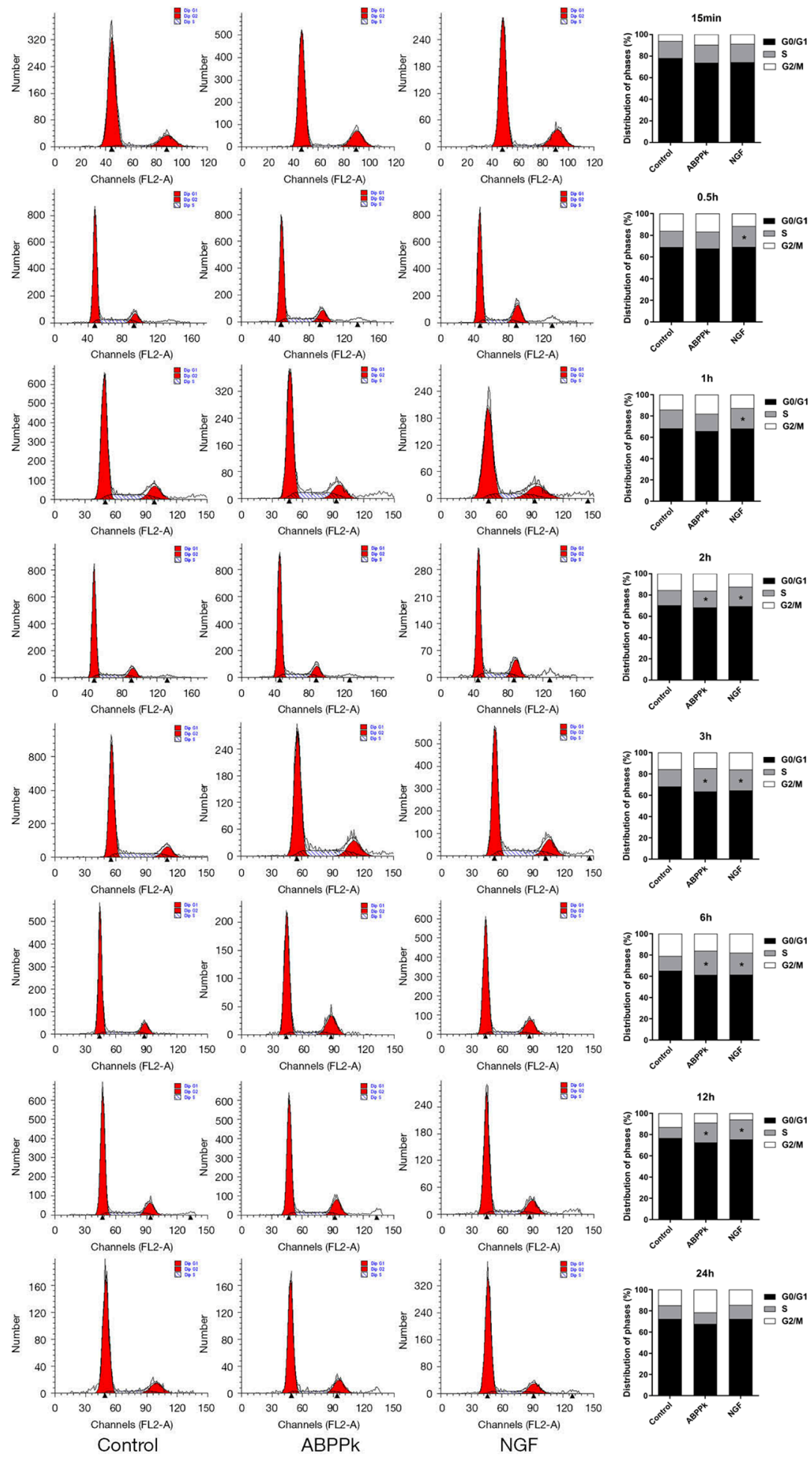

Figure 2 Effect of ABPPk on cell cycle progression of SCs. The cell cycle analysis of SCs was conducted by flow cytometry. ABPPk promotes the proliferation of SCs and transition from G0/G1 to S phase. The distribution of the cell cycle is shown in the graphs. Results are expressed as the mean $\pm \mathrm{SEM}, \mathrm{n}=5 .{ }^{*}, \mathrm{P}<0.05$ vs. the control cells. ABPPk, Achyranthes bidentata polypeptide k; SCs, Schwann cells; SEM, standard error of the mean; NGF, nerve growth factor. 
A
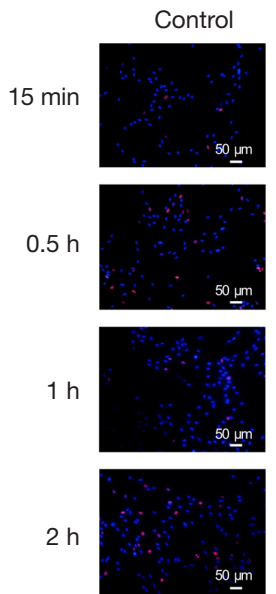
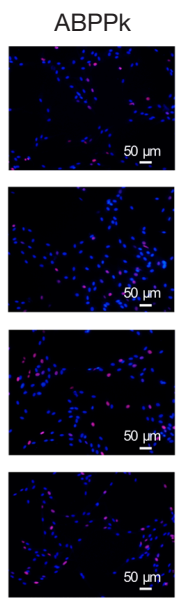
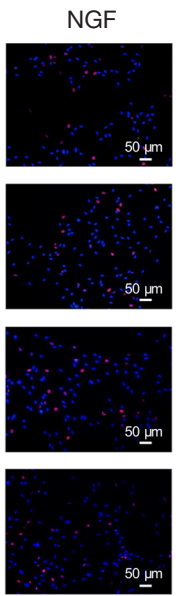
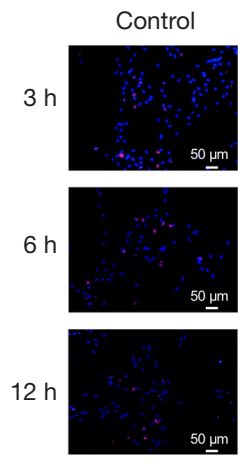

$24 \mathrm{~h}$

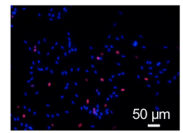

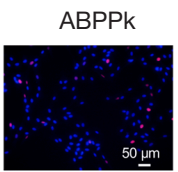
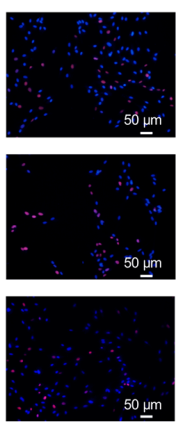
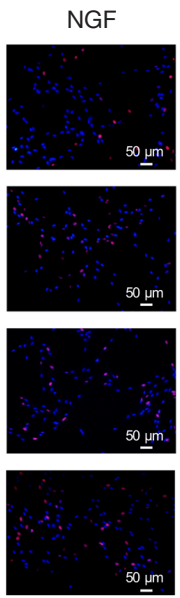

B

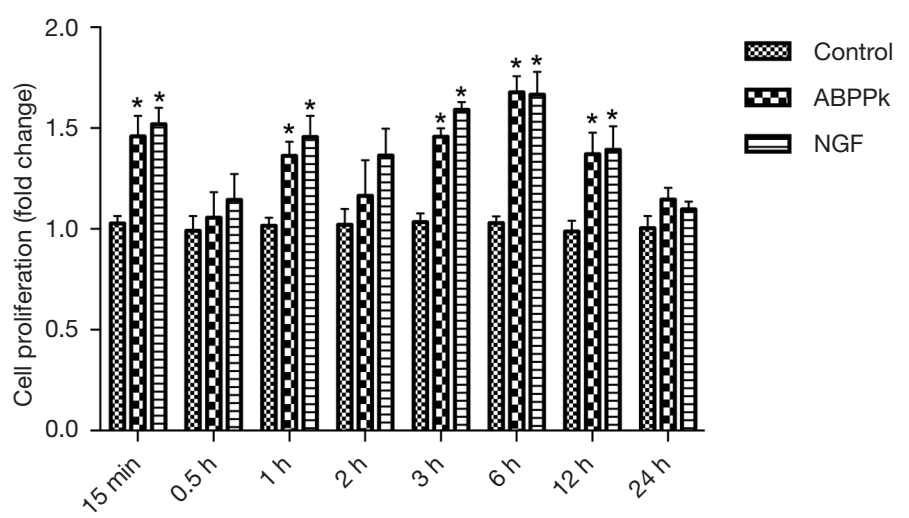

Figure $3 \mathrm{EdU}$ incorporation reflecting the proliferation of SCs. An EdU cell proliferation assay kit was used to assess proliferation. (A) After ABPPk and NGF were treated for different times, SCs were stained with EdU (red) and Hoechst 33342 (blue), and their morphology was confirmed by fluorescence microscope. Scale bar $=50 \mu \mathrm{m}$. (B) The ratio of SCs stained by EdU in each group. Results are expressed as the mean \pm SEM, $n=5$. * $\mathrm{P}<0.05$ vs. the control cells. SCs, Schwann cells; ABPPk, Achyranthes bidentata polypeptide k; NGF, nerve growth factor; SEM, standard error of the mean.

\section{Functional enrichment analysis of genes within the $A B P P k$ group}

In addition to the previously analyzed SC-related functions, we conducted an overall analysis of the biological functions of all genes at each time point, and it was found that the ABPPk group and NGF group still had significant differences in the functions involved. For the ABPPk group, the most enriched GO terms were "response to hypoxia, regulation of axon extension, vascular endothelial growth factor production, regulation of neuron migration, regulation of tissue remodeling, blood vessel lumenization" (Figure 8A), while these were not observed in the NGF group. For the NGF group, the most enriched GO term was "regulation of myelination, axon ensheathment, neurotransmitter receptor metabolic process" (Figure $8 B$ ), while commonality between the ABPPk and NGF groups was seen with "response to ATP, regeneration, negative regulation of neuron apoptotic process, and cell morphogenesis involved in neuron differentiation".

Effect of ABPPk on the gene expression of SCs proliferation in the process of peripheral nerve injury and repair

To observe whether ABPPk had a certain promoting effect on SC proliferation during the repair of sciatic nerve injury, the proteins extracted from the clamped tissue at different time points after the operation were detected by Western blot. The results showed that the ABPPk group 

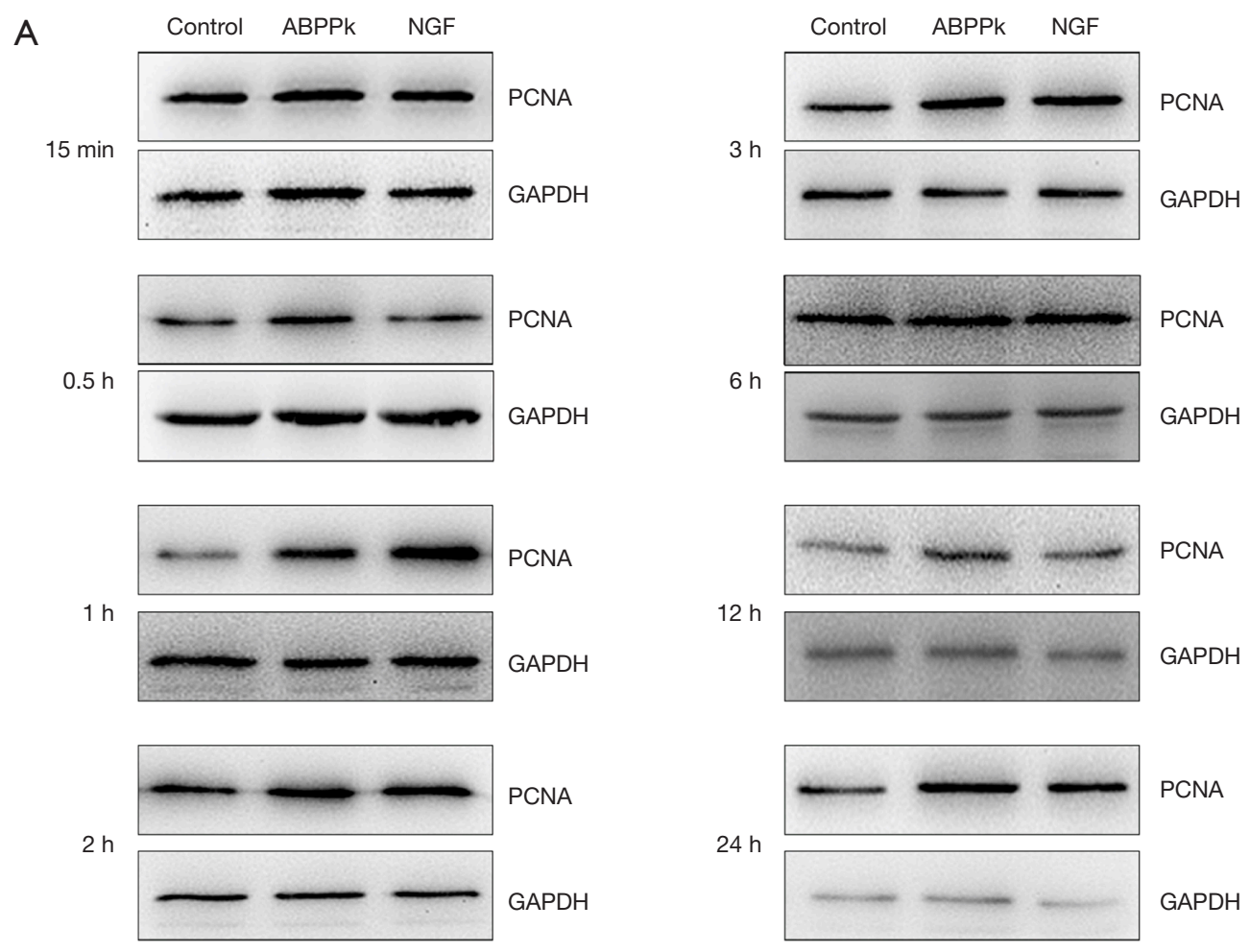

B

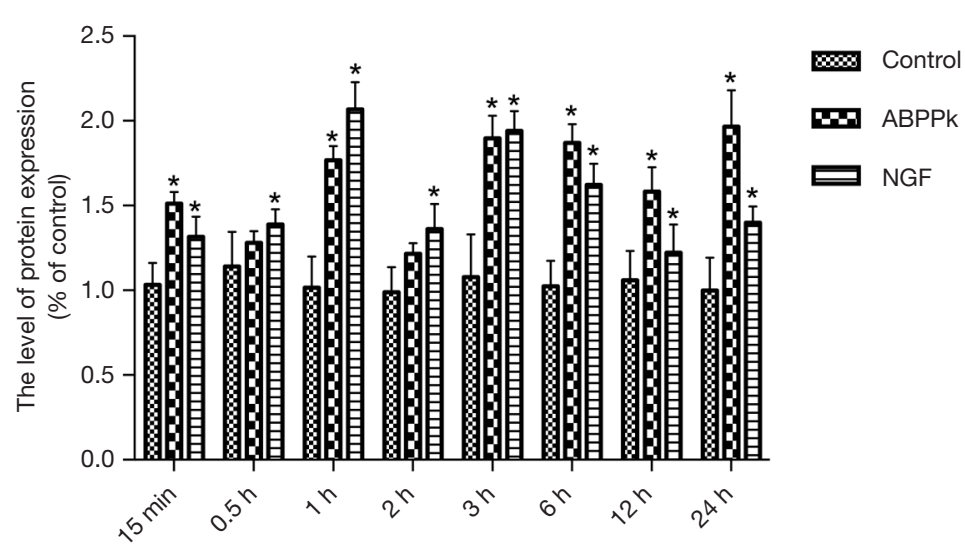

Figure 4 Effect of ABPPk on the expression levels of PCNA. (A) The protein expression of PCNA was measured by using Western blot in SCs after treatment with ABPPk and NGF for different times. (B) Statistical analysis of (A). GAPDH was used as a loading control. Results are expressed as the mean $\pm \mathrm{SEM}, \mathrm{n}=5$. * $\mathrm{P}<0.05$ vs. the control cells. ABPPk, Achyranthes bidentata polypeptide k; PCNA, proliferating cell nuclear antigen; SCs, Schwann cells; NGF, nerve growth factor; SEM, standard error of the mean.

could increase the protein expression of PCNA at 1, 4, and especially at $7 \mathrm{~d}$ after surgery (Figure 9).

\section{Discussion}

The repair of peripheral nerve damage has always been a research hotspot in the field of neuroscience. After peripheral nerve injury, proliferated SCs migrate along the basement membrane to the damaged segment to form the Büngner area, and secrete nutritional factors to guide the axon to grow distally (17-19). SCs can also promote the process of myelination after peripheral nerve injury $(20,21)$, which is very important for the timely and accurate transmission of information between the brain and body 

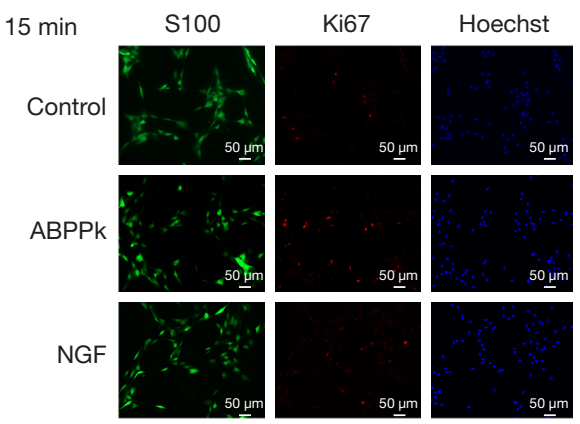

$3 \mathrm{~h}$
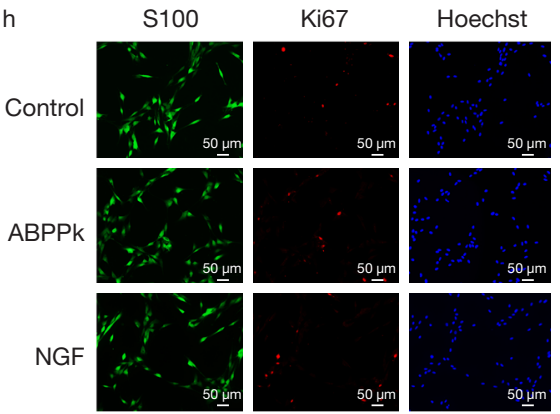
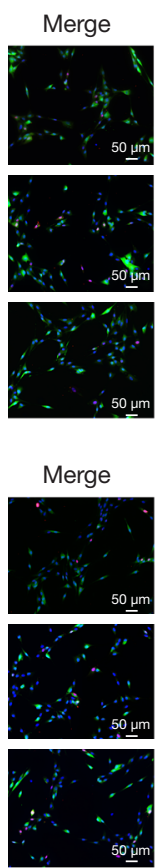

$6 \mathrm{~h}$

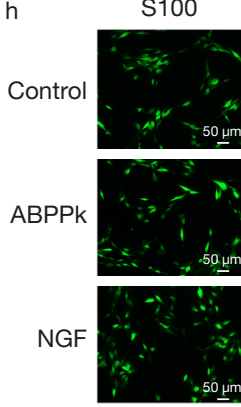

$12 \mathrm{~h}$

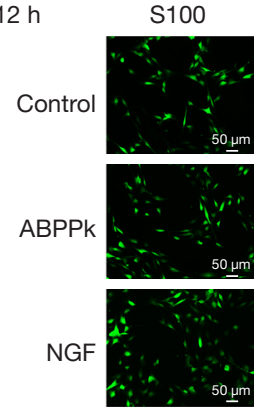

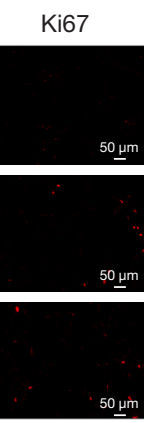
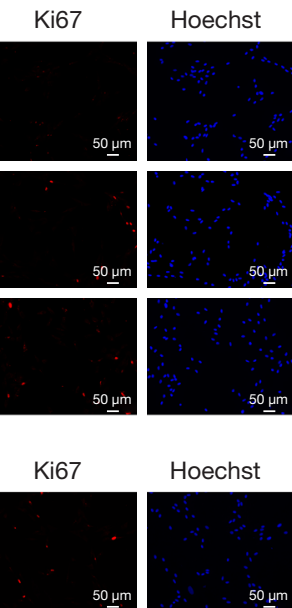

Hoechst
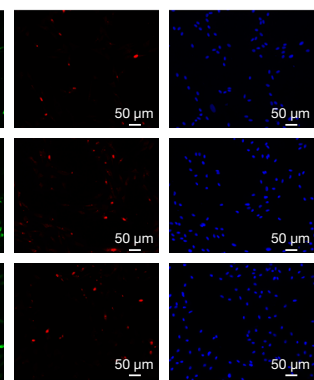

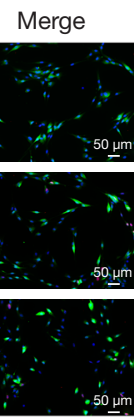

Merge
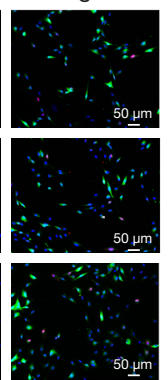

Figure 5 Effect of ABPPk on the expression levels of Ki67. Ki67 staining assay determined the effect of ABPPk and NGF on cell proliferation. Ki67 staining indicated a higher Ki67 positive cell proportion in ABPPk or NGF treated cells for different times (15 min, 3, 6, and $12 \mathrm{~h})$. Scale bar =50 $\mu \mathrm{m}$. ABPPk, Achyranthes bidentata polypeptide k; NGF, nerve growth factor.

parts (22). Therefore, promoting the proliferation of SCs contributes to the repair and functional recovery of peripheral nerves.

Achyranthes bidentata is a well-known traditional Chinese medicine with many functions and effects. In this study, we found that $\mathrm{ABPPk}$ could significantly promote the proliferation of primary SCs cultured in vitro at different times, and to analyze this effect in vivo, we constructed a sciatic nerve injury model. Western blot analysis showed that the proliferation of SCs in the ABPPk and NGF groups was significantly increased compared with the normal saline group, which further indicated that ABPPk could promote the proliferation of SCs. In view of these findings, we suspected that the effect of ABPPk on SC proliferation was regulated by a complex mechanism and revealed this by whole-transcriptome sequencing technology combined with bioinformatics analysis of the IPA database and other methods. Compared with the NGF group, WGCNA analysis showed that $\mathrm{ABPPk}$ was mainly involved in the positive regulation of cell proliferation and the epigenetic regulation module of cell proliferation. The modules and genes involved in ABPPk were significantly different from those of NGF.
In addition to the advantage of promoting SCs proliferation, ABPPk also demonstrated its effect on other functions of SCs. IPA analysis and PPI found that ABPPk also differed from NGF in the biological processes of SC migration, differentiation, movement, and development in terms of action time and key genes. To further explore the effects of ABPPk and NGF on SCs, functional enrichment analysis showed that $\mathrm{ABPPk}$ had more participation and advantages in axonal extension and vascular system, especially in vascular regulation, which was consistent with the original Chinese medicine application and previous research results. Our research group previously conducted a deeper study on the protective effect of ABPPk on serum-deprived SCs through high-throughput sequencing technology, and found that in ABPPk protected SCs, transcription factors were activated earlier and involved in vascular related regulation for a longer time and a wider range of regulation (23). In addition, combined with the WGCNA method to analyze the ABPPk in this study, it is found that one of the modules is defense signal, and many genes in this module are closely related to hypoxia injury protection and promotion of angiogenesis.

Few new plant active peptide drugs have been used in 

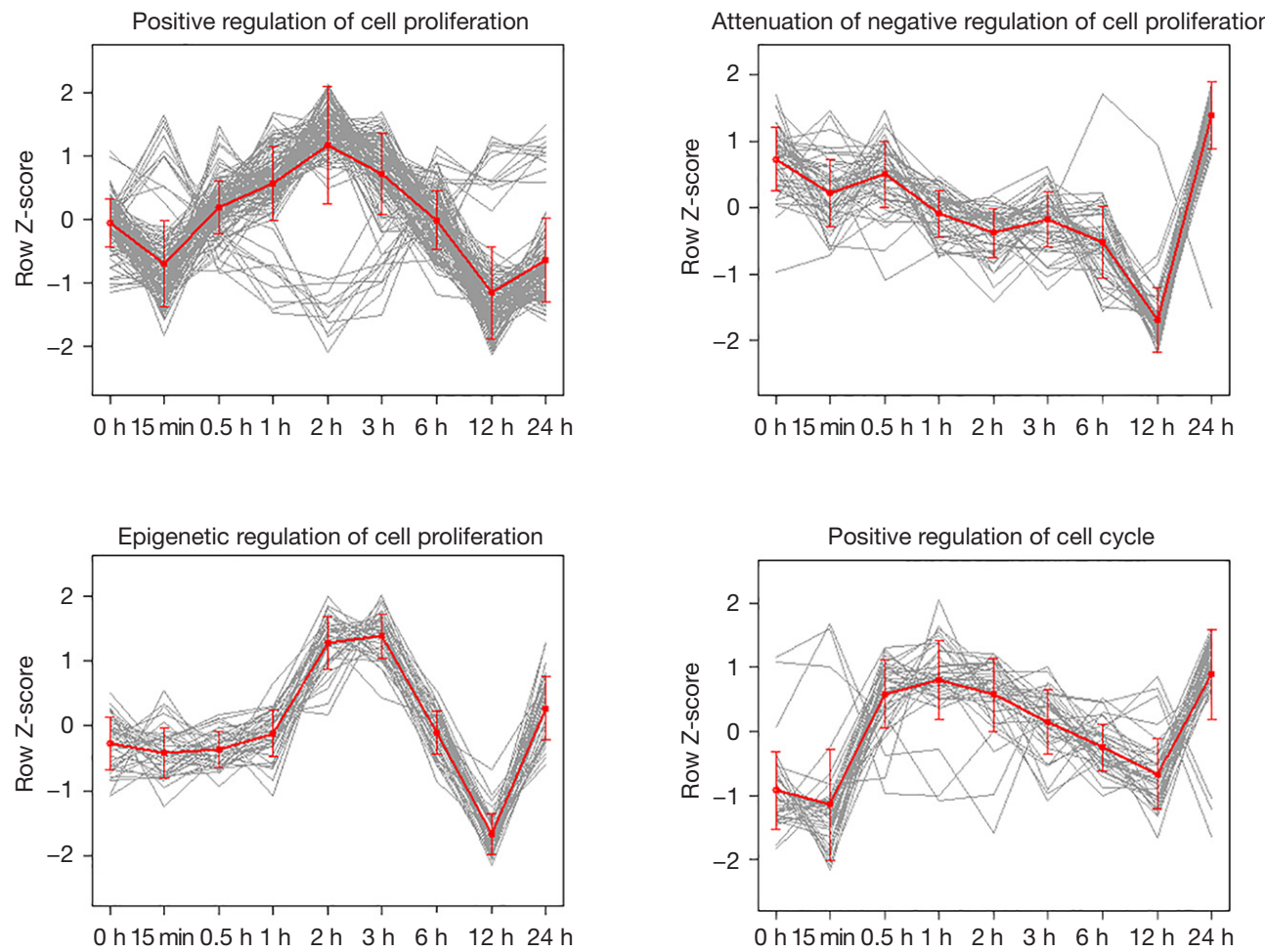

Figure 6 Co-expression modules identified and characterized by WGCNA. The gene expression trend of the different modules of the ABPPk group and the NGF group. The red line indicates the average expression value. WGCNA, weighted gene co-expression network analysis; ABPPk, Achyranthes bidentata polypeptide k; NGF, nerve growth factor.

the clinical treatment of peripheral nerve injury. According to our study, ABPPk can promote SC proliferation early and effectively, and efficiently regulate vascular and axonal extension, ultimately improve peripheral nerve regeneration and quality of life. We also focused on the correlation and difference of ABPPk and NGF to verify their different molecular mechanisms on SCs, and found that compared with existing neurotrophic factors, Achyranthes bidentata from plants has sufficient sources and pharmacological advantages (24). As for a single component, it should not fully represent the role of traditional Chinese medicine, which is still quite complicated. Our research can represent part of the effects of traditional Chinese medicine, so as to provide a target basis for the protective effect of traditional Chinese medicine on peripheral nerve injury. In the future, it will be more recommended to use Chinese herbal medicine Achyranthes bidentata or its active ingredient ABPPk to treat patients with peripheral nerve injury. In summary, our work helps to enhance the understanding of the function and mechanism of $\mathrm{ABPPk}$ and provides an experimental and theoretical basis for the clinical treatment of peripheral nerve injury and the discovery of new therapeutic targets. 


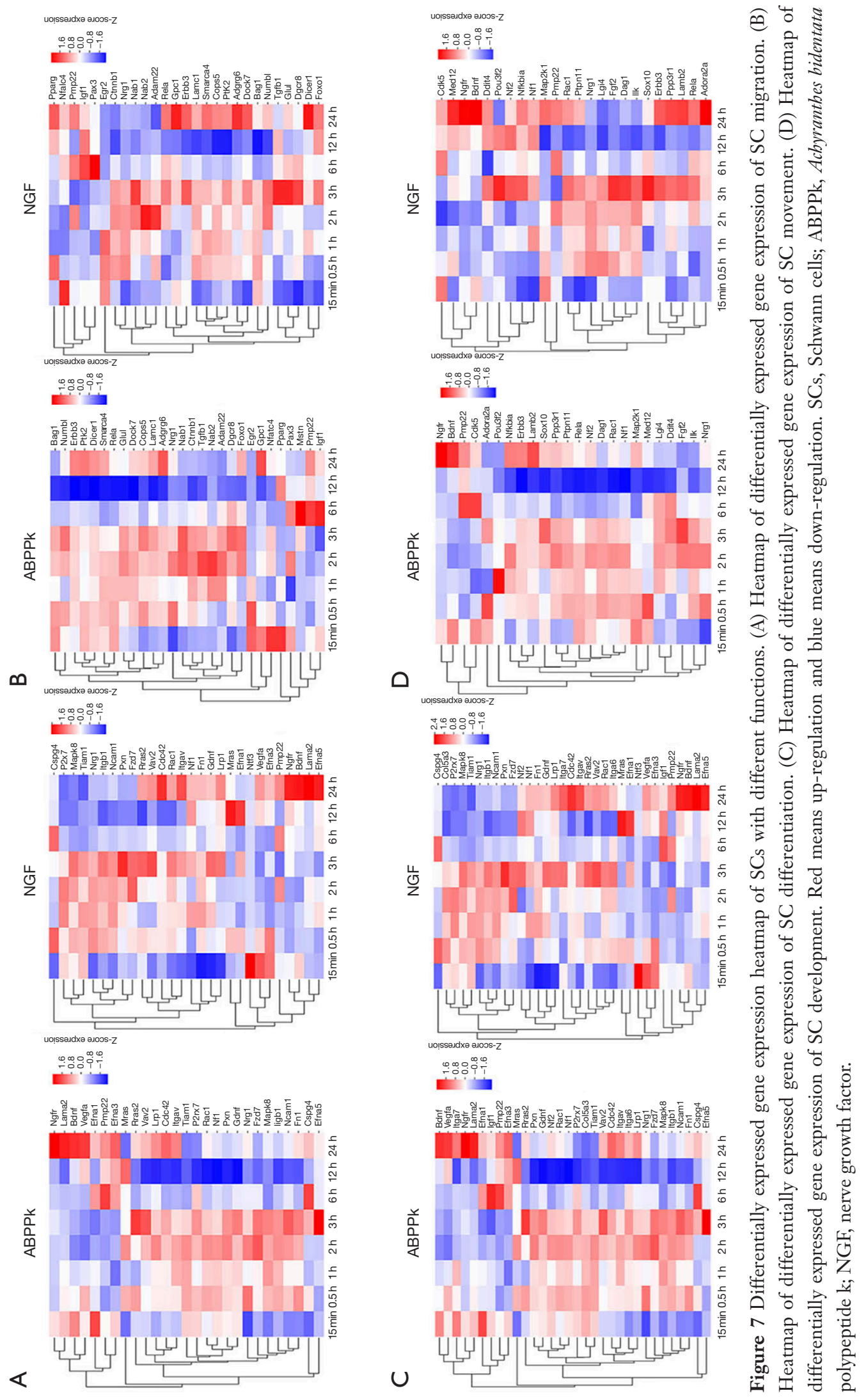


A

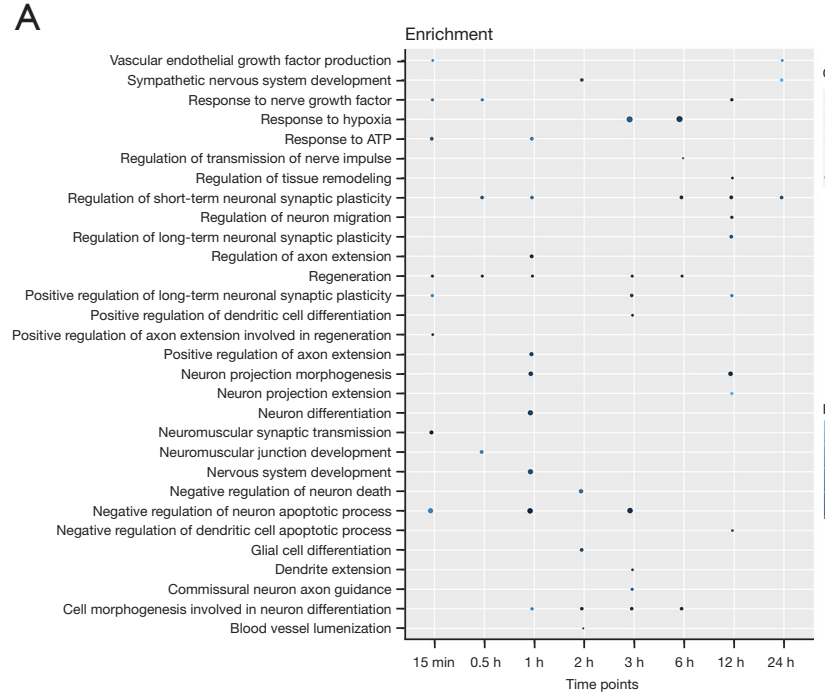

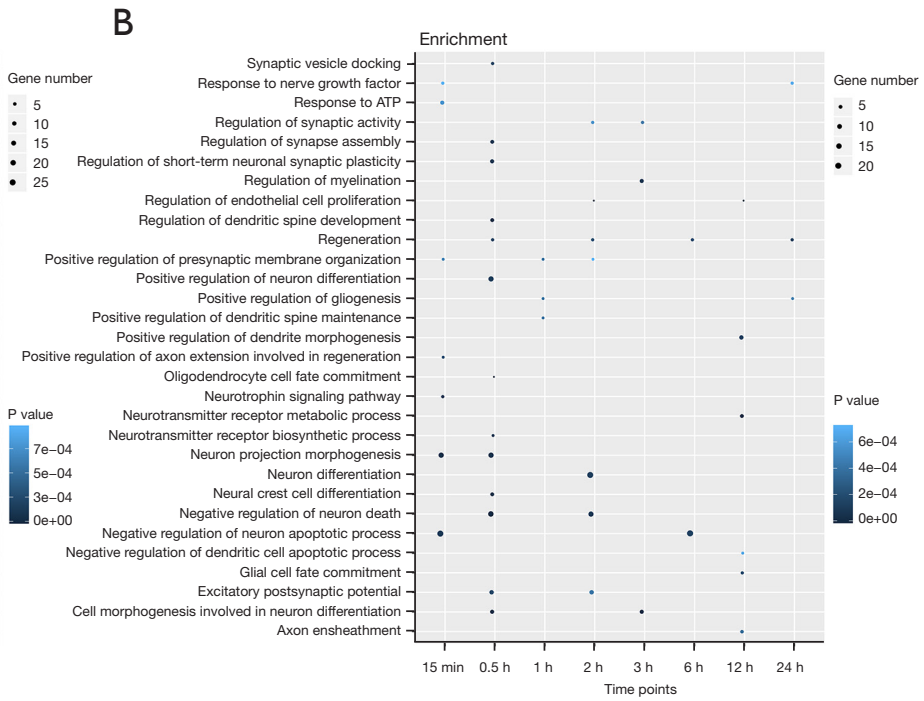

Figure 8 GO enrichment analysis of genes. (A) The GO enrichment analysis in the ABPPk group. (B) The GO enrichment analysis in the NGF group. The number of differentially expressed gene annotated in the specific GO was expressed as the size of the circle. GO, gene ontology; ABPPk, Achyranthes bidentata polypeptide k; NGF, nerve growth factor.

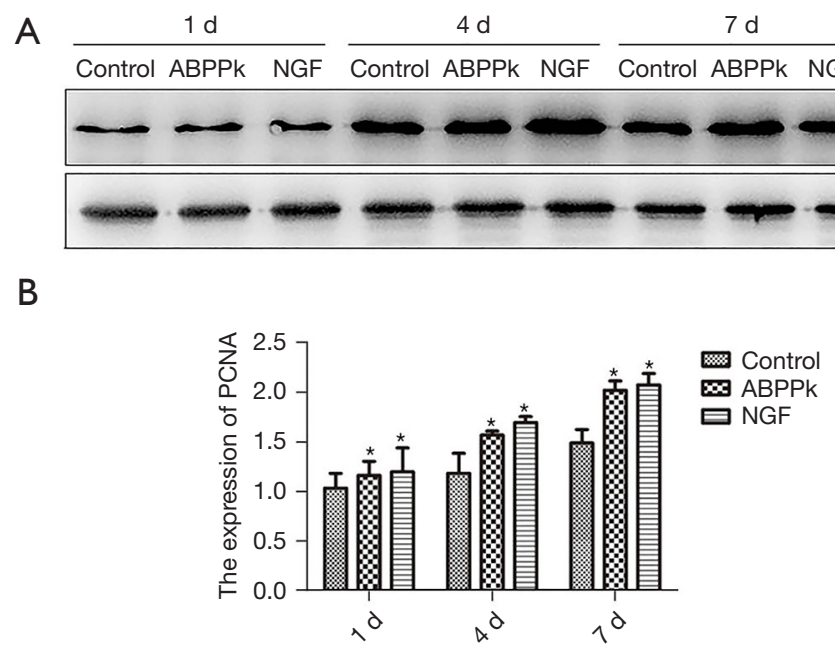

Figure $9 \mathrm{Effect}$ of ABPPk on the protein expression of SC proliferation in the process of peripheral nerve injury and repair. (A) Western blot analysis indicated that both ABPPk and NGF could increase the expression of PCNA protein after sciatic nerve injury at different times $(1,4$, and 7 d). (B) Statistical analysis of (A). GAPDH was used as a loading control. Results are expressed as the mean \pm SEM, $n=5$. *,$P<0.05$ vs. the control cells. ABPPk, Achyranthes bidentata polypeptide k; SC, Schwann cell; NGF, nerve growth factor; PCNA, proliferating cell nuclear antigen; SEM, standard error of the mean.

\section{Acknowledgments}

Funding: This work was supported by grants from the National Natural Science Foundation of China (Grant No. 81901256), the Basic Research Project of the Jiangsu Education Department (Grant No. 18KJD310002, 19KJD310001), and Jiangsu Provincial Double-Innovation Doctor Program (JSSCBS20211095). 


\section{Footnote}

Reporting Checklist: The authors have completed the ARRIVE reporting checklist. Available at https://dx.doi. org/10.21037/atm-21-5181

Data Sharing Statement: Available at https://dx.doi. org/10.21037/atm-21-5181

Conflicts of Interest: All authors have completed the ICMJE uniform disclosure form (available at https://dx.doi. org/10.21037/atm-21-5181). The authors have no conflicts of interest to declare.

Ethical Statement: The authors are accountable for all aspects of the work in ensuring that questions related to the accuracy or integrity of any part of the work are appropriately investigated and resolved. Experiments were performed under a project license (No. 2019030315) granted by the Laboratory Animal Ethics Committee of Nantong University, in compliance with the Nantong University institutional guidelines for the care and use of animals.

Open Access Statement: This is an Open Access article distributed in accordance with the Creative Commons Attribution-NonCommercial-NoDerivs 4.0 International License (CC BY-NC-ND 4.0), which permits the noncommercial replication and distribution of the article with the strict proviso that no changes or edits are made and the original work is properly cited (including links to both the formal publication through the relevant DOI and the license). See: https://creativecommons.org/licenses/by-nc-nd/4.0/.

\section{References}

1. Raza C, Riaz HA, Anjum R, et al. Repair strategies for injured peripheral nerve: Review. Life Sci 2020;243:117308.

2. Zhu H, Xue C, Yao M, et al. miR-129 controls axonal regeneration via regulating insulin-like growth factor-1 in peripheral nerve injury. Cell Death Dis 2018;9:720.

3. Ko KR, Lee J, Lee D, et al. Hepatocyte growth factor (HGF) promotes peripheral nerve regeneration by activating repair Schwann cells. Sci Rep 2018;8:8316.

4. Reed CB, Frick LR, Weaver A, et al. Deletion of calcineurin in Schwann cells does not affect developmental myelination, but reduces autophagy and delays myelin clearance after peripheral nerve injury. J Neurosci 2020;40:6165-76.

5. Poplawski G, Ishikawa T, Brifault C, et al. Schwann cells regulate sensory neuron gene expression before and after peripheral nerve injury. Glia 2018;66:1577-90.

6. Chen J, Ren S, Duscher D, et al. Exosomes from human adipose-derived stem cells promote sciatic nerve regeneration via optimizing Schwann cell function. J Cell Physiol 2019;234:23097-110.

7. Cheng Q, Tong F, Shen Y, et al. Achyranthes bidentata polypeptide $\mathrm{k}$ improves long-term neurological outcomes through reducing downstream microvascular thrombosis in experimental ischemic stroke. Brain Res 2019;1706:166-76.

8. Hua S, Zhang X. Effects of Achyranthes bidentata alcohol on proliferation capacity of osteoblasts and miRNA in Runx2. Exp Ther Med 2019;18:1545-50.

9. Peng S, Wang C, Ma J, et al. Achyranthes bidentata polypeptide protects dopaminergic neurons from apoptosis in Parkinson's disease models both in vitro and in vivo. $\mathrm{Br}$ J Pharmacol 2018;175:631-43.

10. Li M, Zhu Y, Peng W, et al. Achyranthes bidentata polypeptide protects Schwann cells from apoptosis in hydrogen peroxide-induced oxidative stress. Front Neurosci 2018;12:868.

11. Chen J, Li C, Liu W, et al. miRNA-155 silencing reduces sciatic nerve injury in diabetic peripheral neuropathy. $\mathrm{J}$ Mol Endocrinol 2019;63:227-38.

12. Hu Y, Deng J, Tian K, et al. MiR-8-3p regulates hyperthermia-induced lactate secretion by targeting PPP2R5B in boar Sertoli cells. Mol Reprod Dev 2019;86:1720-30.

13. Cheng Q, Jiang C, Wang C, et al. The Achyranthes bidentata polypeptide $\mathrm{k}$ fraction enhances neuronal growth in vitro and promotes peripheral nerve regeneration after crush injury in vivo. Neural Regen Res 2014;9:2142-50.

14. Zhong J, Zhou J, Sun H, et al. Effects of Salvia miltiorrhiza injection on apoptosis of Schwann cells induced by hydrogen peroxide. Ann Palliat Med 2021;10:625-32.

15. Xu Y, Geng R, Yuan F, et al. Identification of differentially expressed key genes between glioblastoma and low-grade glioma by bioinformatics analysis. PeerJ 2019;7:e6560.

16. Yu G, Wang LG, Han Y, et al. clusterProfiler: an R package for comparing biological themes among gene clusters. OMICS 2012;16:284-7.

17. Jessen KR, Mirsky R. The repair Schwann cell and its function in regenerating nerves. J Physiol 2016;594:3521-31. 
18. Maugeri G, D'Amico AG, Musumeci G, et al. Effects of Pacap on Schwann cells: focus on nerve injury. Int $\mathrm{J}$ Mol Sci 2020;21:8233.

19. Panzer KV, Burrell JC, Helm KVT, et al. Tissue engineered bands of Büngner for accelerated motor and sensory axonal outgrowth. Front Bioeng Biotechnol 2020;8:580654.

20. Choi SJ, Park SY, Shin YH, et al. Mesenchymal stem cells derived from Wharton's Jelly can differentiate into Schwann cell-like cells and promote peripheral nerve regeneration in acellular nerve grafts. Tissue Eng Regen Med 2021;18:467-78.

21. Nocera G, Jacob C. Mechanisms of Schwann cell plasticity involved in peripheral nerve repair after injury. Cell Mol Life Sci 2020;77:3977-89.

Cite this article as: Tang L, Zhang M, Liu X, Zhu Y, Chen X, Zhong J, Li M. Effects and molecular mechanisms of Achyranthes bidentata polypeptide k on proliferation of Schwann cells. Ann Transl Med 2021;9(20):1581. doi: 10.21037/atm-215181
22. Cawley JL, Jordan LR, Wittenberg NJ. Detection and characterization of vesicular gangliosides binding to myelin-associated glycoprotein on supported lipid bilayers. Anal Chem 2021;93:1185-92.

23. Li M, Zhu Y, Tang L, et al. Protective effects and molecular mechanisms of Achyranthes bidentata polypeptide k on Schwann cells. Ann Transl Med 2021;9:381.

24. Yan C, Zhang S, Wang C, et al. A fructooligosaccharide from Achyranthes bidentata inhibits osteoporosis by stimulating bone formation. Carbohydr Polym 2019;210:110-8.

(English Language Editor: B. Draper) 


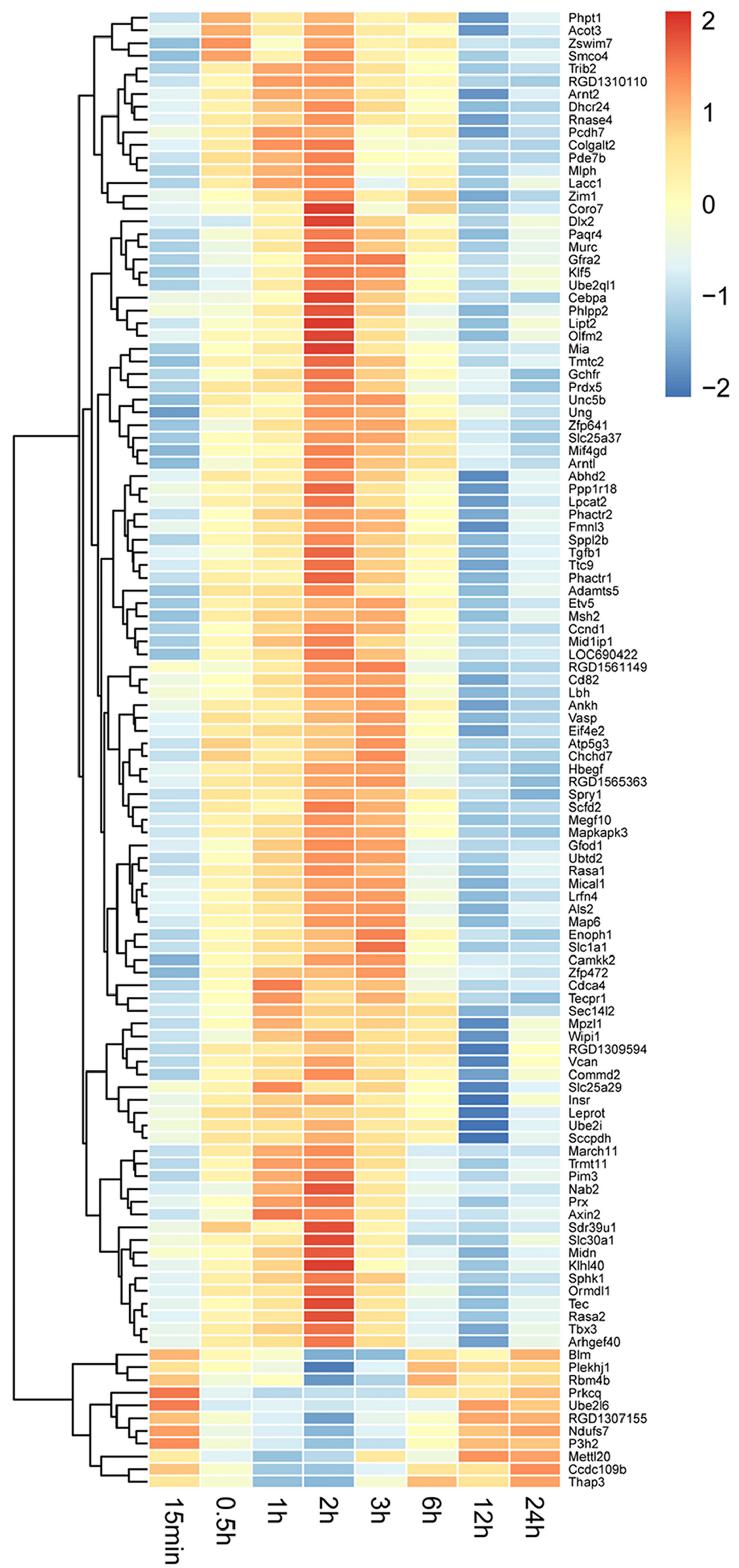

Figure S1 The heatmap of hub genes in the positively regulates cell proliferation module of the ABPPk group identified by WGCNA. WGCNA, weighted gene co-expression network analysis; ABPPk, Achyranthes bidentata polypeptide k. 


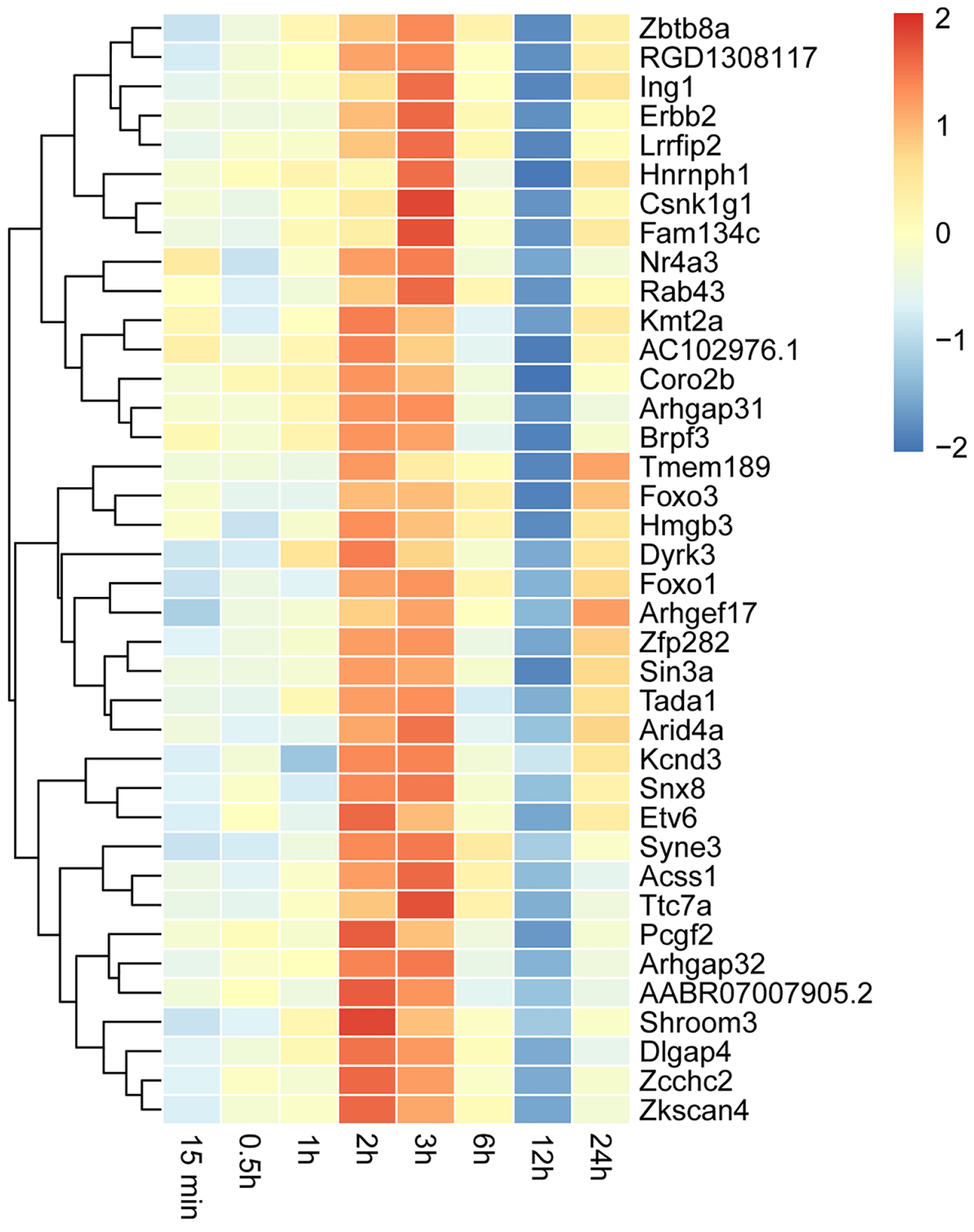

Figure S2 The heatmap of hub genes in the epigenetic regulation of cell proliferation module of the ABPPk group identified by WGCNA. ABPPk, Achyranthes bidentata polypeptide k; WGCNA, weighted gene co-expression network analysis. 


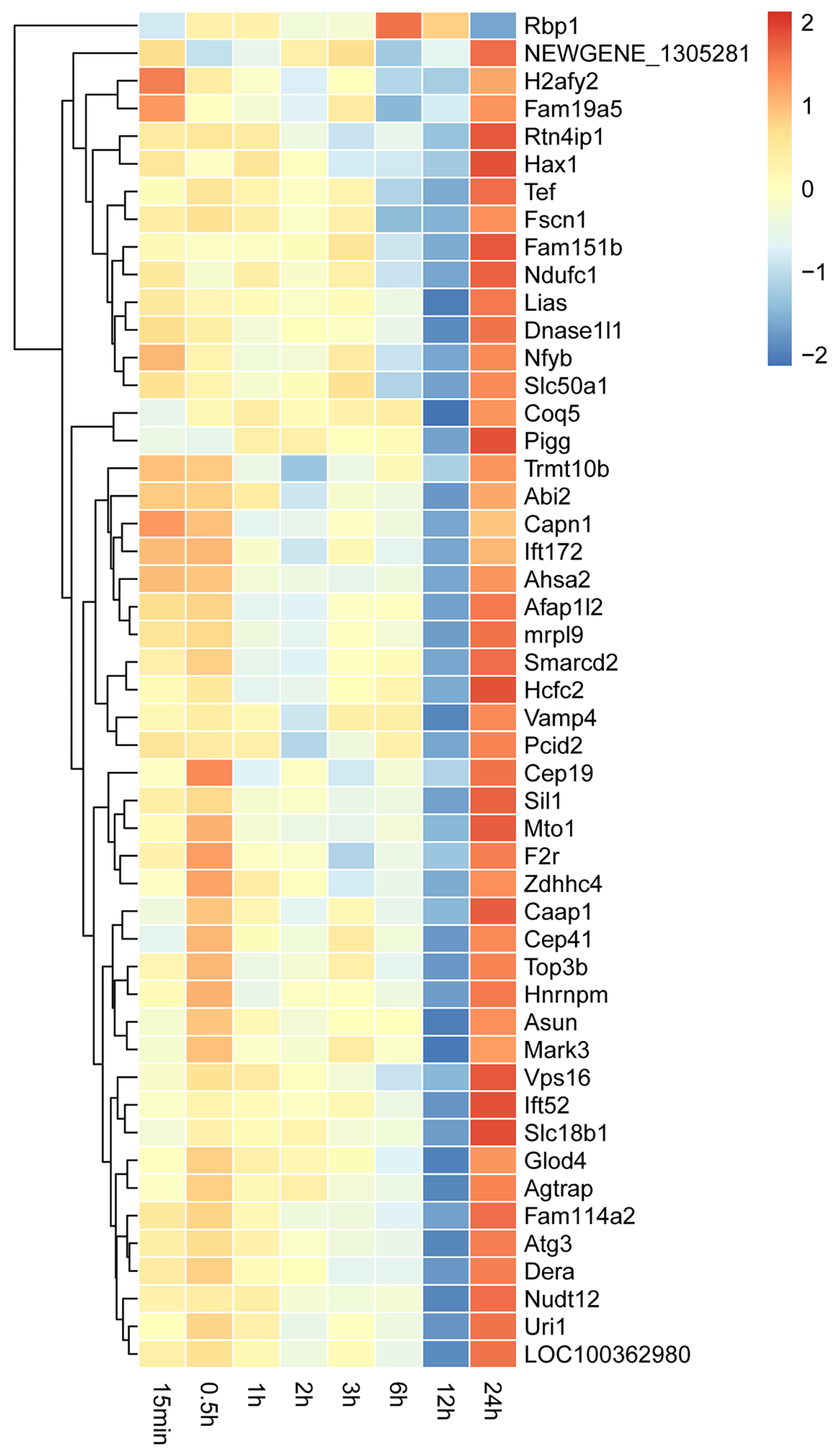

Figure S3 The heatmap of hub genes in the attenuation of negative regulation of cell proliferation module of the NGF group identified by WGCNA. NGF, nerve growth factor; WGCNA, weighted gene co-expression network analysis. 


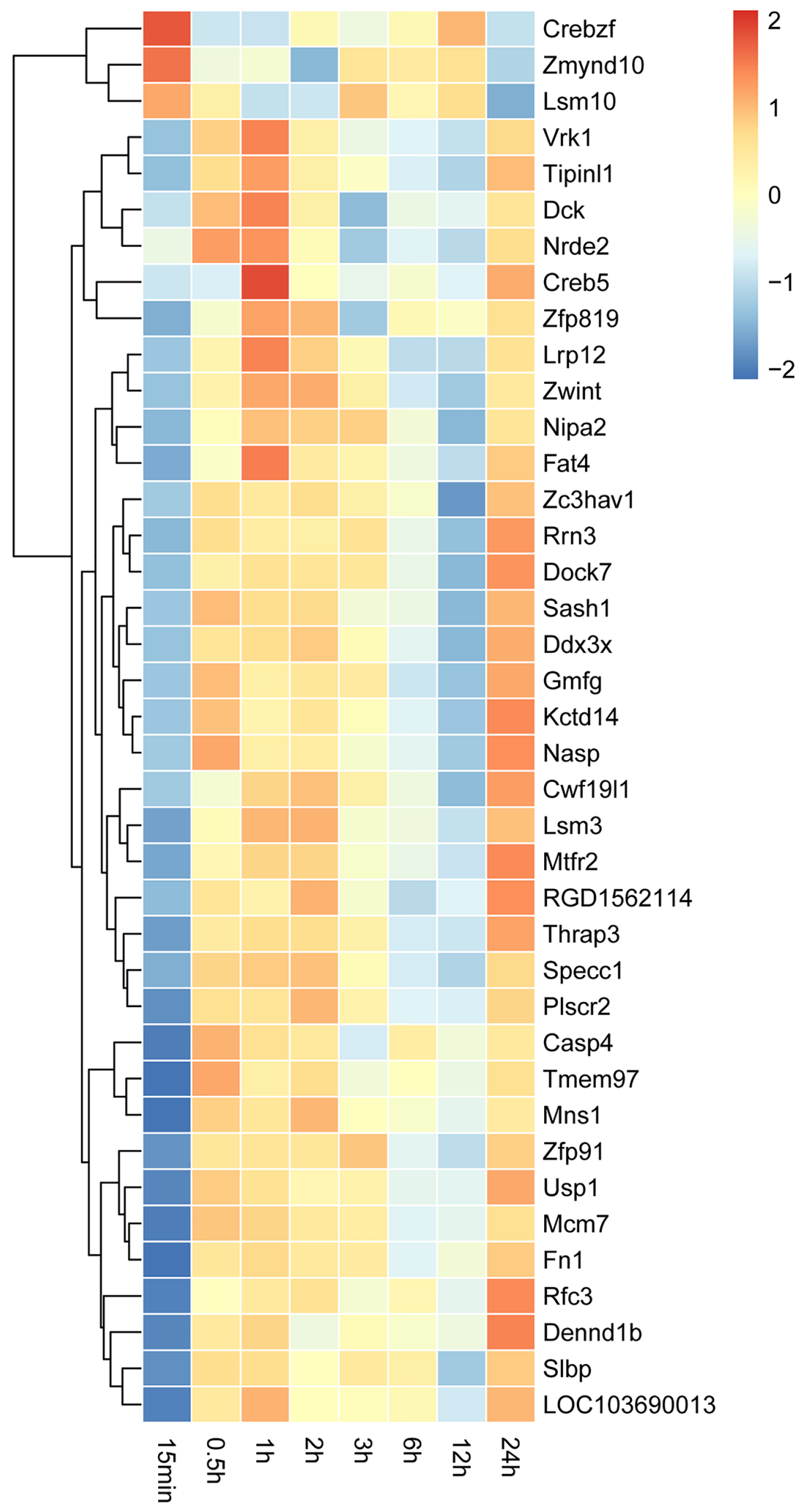

Figure S4 The heatmap of hub genes in the positive regulation of cell cycle module of the NGF group identified by WGCNA. NGF, nerve growth factor; WGCNA, weighted gene co-expression network analysis. 
A

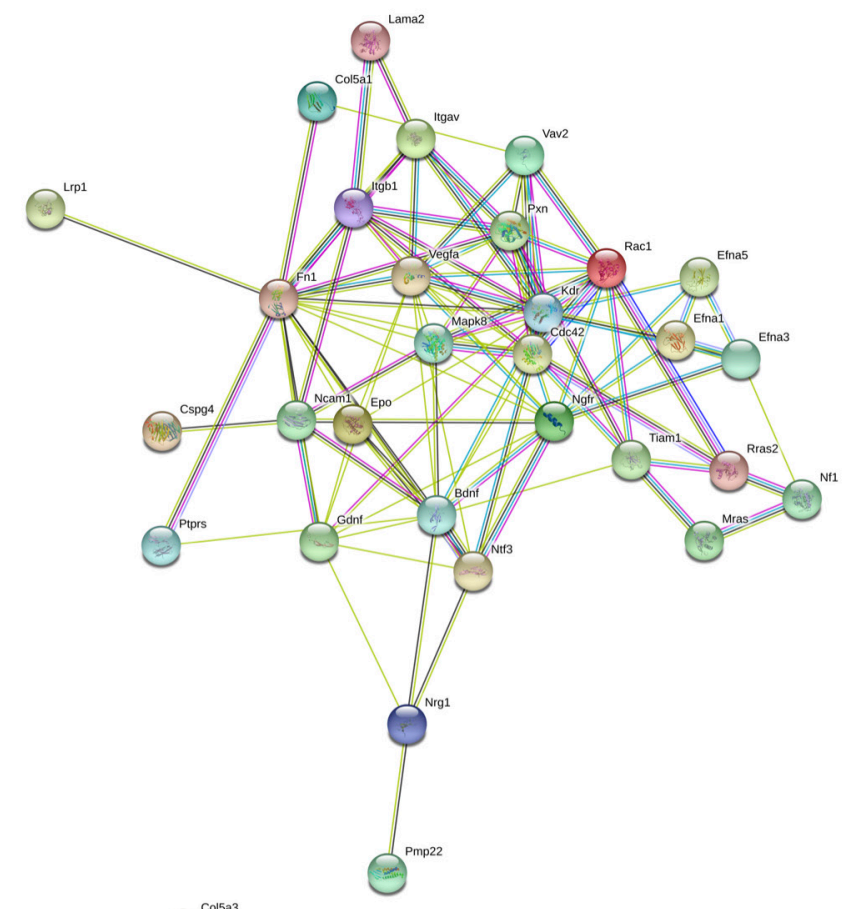

C

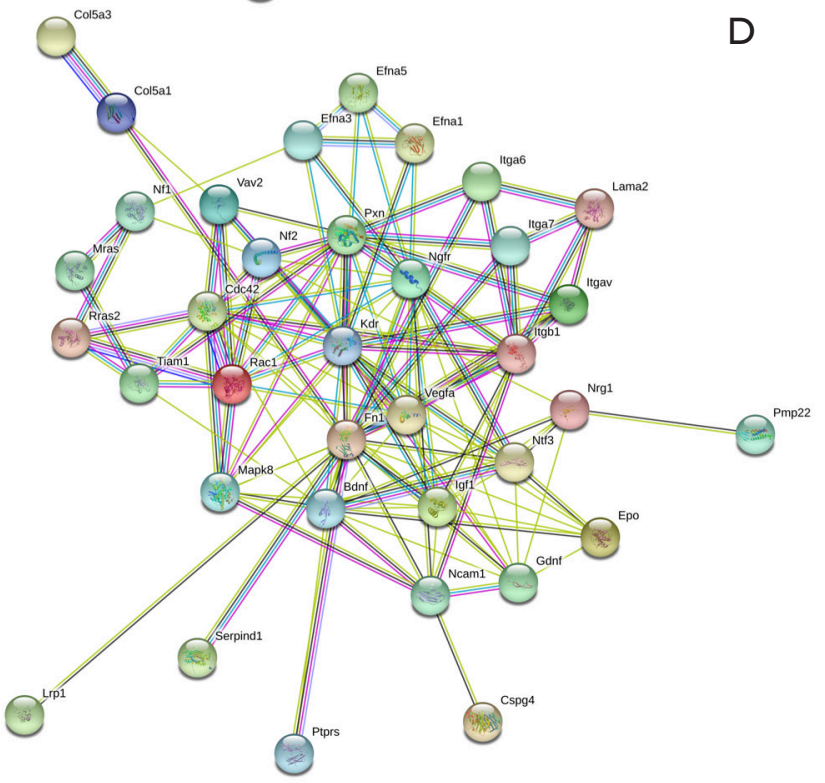

B
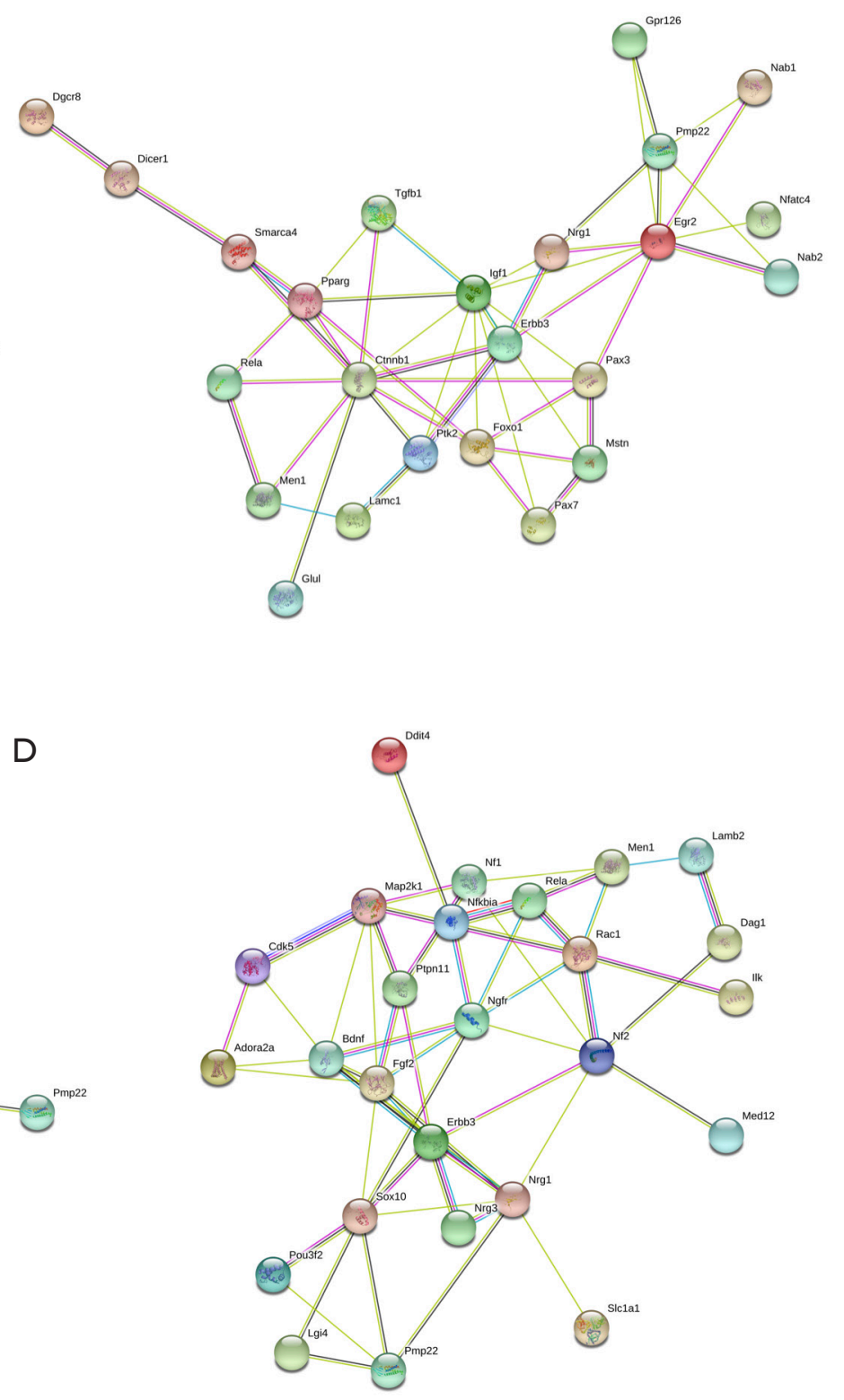

Figure S5 The PPI networks of hub genes of different functions. (A) The PPI network of the hub genes of SC migration. (B) The PPI network of the hub genes of SC differentiation. (C) The PPI network of the hub genes of SC movement. (D) The PPI network of the hub genes of SC development. PPI, protein-protein interaction; SC, Schwann cell. 